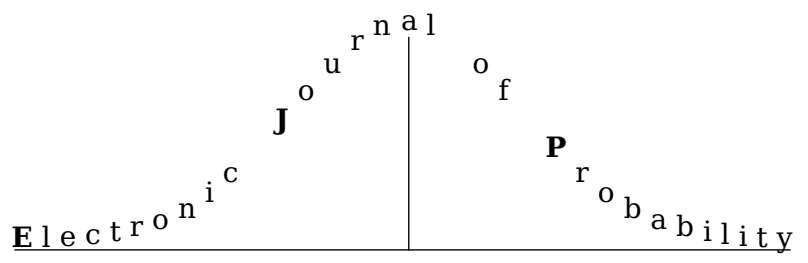

Electron. J. Probab. 26 (2021), article no. 138, 1-17.

ISSN: 1083-6489 https://doi.org/10.1214/21-EJP681

\title{
Averaging in the case of multiple invariant measures for the fast system
}

\author{
M. Freidlin* $\quad$ L. Koralov ${ }^{\dagger}$
}

\begin{abstract}
We consider the averaging principle for deterministic and stochastic systems with a fast stochastic component (family of continuous time Markov chains depending on the state of the system as a parameter). We show that, due to the nontrivial structure of the simplex of invariant probability measures of the chains, the limiting system should be considered on a graph with certain gluing conditions at the vertices of the graph.

Keywords: fast-slow system; averaging; simplex of invariant measures; gluing conditions; processes on graphs.

MSC2020 subject classifications: 70K70; 70K65; 35B40; 34C29.

Submitted to EJP on February 24, 2020, final version accepted on July 31, 2021.
\end{abstract}

\section{Introduction}

Consider the $d$-dimensional continuous stochastic process $z_{t}^{\varepsilon}$ satisfying the equation

$$
d z_{t}^{\varepsilon}=v\left(\xi_{t}^{\varepsilon}, z_{t}^{\varepsilon}\right) d t+\varkappa d W_{t}, \quad 0<\varepsilon \ll 1 .
$$

We assume that $v$ is sufficiently smooth, and $\xi_{t}^{\varepsilon}=\xi_{t / \varepsilon}$, where $\xi_{t}$ is a stationary process with sufficiently good mixing properties, such as a non-degenerate diffusion on a compact manifold or a continuous time Markov chain on a finite state space (we consider the latter case in this paper). The Wiener process $W_{t}$ is independent of $\xi_{t}^{\varepsilon}$. The coefficient $\varkappa$ is non-negative.

Put $\bar{v}(z)=\operatorname{Ev}\left(\xi_{t}, z\right)$. Then (see, for example, [9] Section 7.2)

$$
z_{t}^{\varepsilon} \rightarrow \bar{z}_{t}, \quad \text { as } \varepsilon \downarrow 0,
$$

(convergence, in distribution, of the processes), where $\bar{z}_{t}$ is the solution of the equation

$$
d \bar{z}_{t}=\bar{v}\left(\bar{z}_{t}\right) d t+\varkappa d W_{t}
$$

*Dept of Mathematics, University of Maryland, College Park, MD 20742.

E-mail: mi f@math.umd .edu

${ }^{\dagger}$ Dept of Mathematics, University of Maryland, College Park, MD 20742.

E-mail: koralov@math.umd.edu 
Averaging in the case of multiple invariant measures for the fast system

with the same initial condition as $z_{t}^{\varepsilon}$. The convergence of $z_{t}^{\varepsilon}$ to $\bar{z}_{t}$ is preserved if the process $\xi_{t}$ is not stationary but converges with probability one to a stationary ergodic process $\tilde{\xi}_{t}$. In this case, $\bar{v}(z)=\operatorname{E} v\left(\tilde{\xi}_{t}, z\right)$. Moreover, the fast component $\xi_{t}^{\varepsilon}$ in (1.1) can depend on the slow component. In order to illustrate this point, let us focus on the case when the fast motion is governed by a continuous time Markov chain $\Xi_{t}^{z}$ on the finite state space $\{1, \ldots, n\}$. The transition rates for the chain $\Xi_{t}^{z}$, which depends on the parameter $z \in \mathbb{R}^{d}$, will be denoted by $q_{i j}(z) \geq 0,1 \leq i, j \leq n, i \neq j$. Intuitively, the slow motion $z_{t}^{\varepsilon}$ is governed, at short time scales, by (1.1) with $\xi_{t}^{\varepsilon}=\xi_{t / \varepsilon}$ replaced by $\Xi_{t / \varepsilon}^{z}$. Yet, we cannot simply say that $\Xi_{t / \varepsilon}^{z}$ is the fast component of the process since $z$ itself evolves (although slowly) in time. The fast-slow system $X_{t}^{\varepsilon}=\left(\xi_{t}^{\varepsilon}, z_{t}^{\varepsilon}\right)$ can be defined constructively (as in Section 2) or by describing its generator. Namely, for $1 \leq i \leq n$, consider the operators

$$
L_{i} u(z)=\frac{\varkappa^{2}}{2} \Delta u(z)+v(i, z) \nabla u(z),
$$

where $u$ is a function defined on $\mathbb{R}^{d}$. These operators would govern the evolution of the slow component for the fixed value $i$ of the fast component in the absence of the fast motion. The second order term, the Laplacian in our case, could also be a more general operator to allow for more general diffusion in the slow variable. To account for the fast component, we define the operator

$$
A^{\varepsilon} f(i, z)=\frac{1}{\varepsilon}\left(\sum_{j \neq i} q_{i j}(z)(f(j, z)-f(i, z))\right)+L_{i} f(i, z),
$$

where $f$ is a function on $\{1, \ldots, n\} \times \mathbb{R}^{d}$. This operator, with the properly specified domain, is the generator of the process $X_{t}^{\varepsilon}=\left(\xi_{t}^{\varepsilon}, z_{t}^{\varepsilon}\right)$.

If $q_{i j}(z)>0$ whenever $i \neq j$, then the process $\Xi_{t}^{z}$ has a unique invariant distribution $\mu(z)=\left(\mu_{1}(z), \ldots, \mu_{n}(z)\right)$, and (1.2) holds with $\bar{v}(z)=\sum_{i=1}^{n} \mu_{i}(z) v(i, z)$. Assume now that there is a closed domain $G$ with a smooth boundary such that the chain $\Xi_{t}^{z}$ is ergodic for $z \notin G$ and has, say, two ergodic components $R_{1}=\{1, \ldots, m\}$ and $R_{2}=$ $\{m+1, \ldots, n\}$ for $z \in G$. Thus the transitions between $R_{1}$ and $R_{2}$ are impossible while $z_{t}^{\varepsilon} \in G$. Then one can expect that, as long as $z_{t}^{\varepsilon}$ remains in $G$, it converges, as $\varepsilon \downarrow 0$, to the solution of (1.3) either with $\bar{v}(z)=\sum_{i \in R_{1}} \mu_{i}(z) v(i, z) / \sum_{i \in R_{1}} \mu_{i}(z)$ or with $\bar{v}(z)=$ $\sum_{i \in R_{2}} \mu_{i}(z) v(i, z) / \sum_{i \in R_{2}} \mu_{i}(z)$, depending on whether the fast component evolves in $R_{1}$ or $R_{2}$. Note that, while the invariant distribution is not determined uniquely for $z \in G$, the above expressions for $\bar{v}$ are.

The process $z_{t}^{\varepsilon}$ can go from $G$ to $\mathbb{R}^{d} \backslash G$ and vice versa in finite time. Therefore, in order to define the limiting process, one should describe the behavior of the process in an infinitesimal neighborhood of $\partial G$. The novelty of the current work is that, in the presence of multiple invariant measures for the fast process, the limiting motion for the slow component is (and needs to be) considered on a graph or an open book (if $d>1$ ), i.e., a more sophisticated space than the case of non-degenerate fast component, where the limiting process lives on the Euclidean space. For simplicity, we will consider the one-dimensional case, where the structure of the simplex of invariant probability measures is already non-trivial.

The phase space of a fast-slow system with one-dimensional slow component is shown in Figure 1. The slow motion takes place in the horizontal direction, with different lines corresponding to the states of the fast variable. The region $G=[0, \infty)$ is where the fast motion is not ergodic. The limiting motion for the slow variable takes place on the graph, denoted by $S$, with one edge (negative semi-axis, denoted by $I_{0}$ ) corresponding to the region where the fast motion is ergodic and two edges (extending to the right, denoted by $I_{1}$ and $I_{2}$ ) encoding the location of the slow component (horizontal direction) and 
Averaging in the case of multiple invariant measures for the fast system
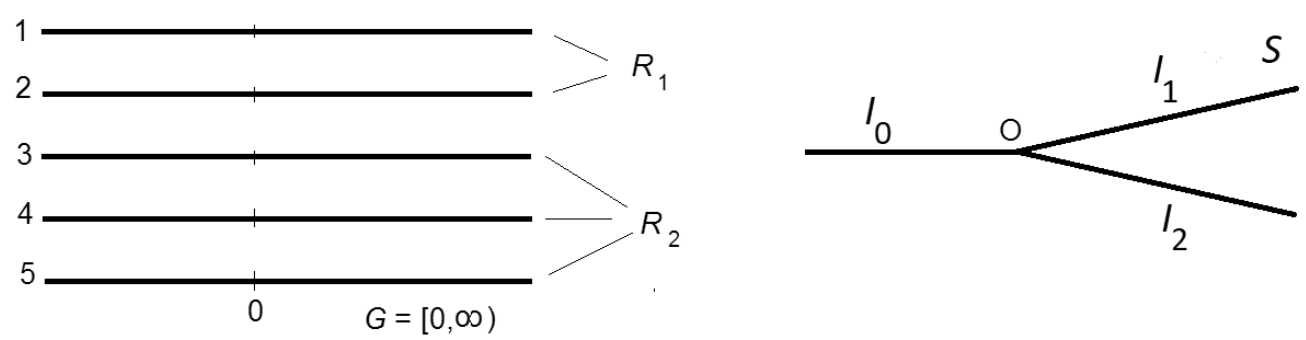

Figure 1: The phase space of the fast-slow system and the corresponding graph. For $z \geq 0$, the chain $\Xi_{t}^{z}$ has two ergodic classes, $R_{1}=\{1,2\}$ and $R_{2}=\{3,4,5\}$. The point $z=0$ corresponds to the vertex $O$ of the graph.

the ergodic component of the Markov chain $\left(R_{1}\right.$ or $\left.R_{2}\right)$ corresponding to the number of the edge. The behavior of the limiting process at the vertex will be specified by the appropriate gluing condition.

Remark. The solutions of the Cauchy problem and of various initial-boundary problems for PDE systems related to the operator $A^{\varepsilon}$ can be written as expectations of certain functionals of the process $X_{t}^{\varepsilon}=\left(\xi_{t}^{\varepsilon}, z_{t}^{\varepsilon}\right)$. This allows one to calculate the asymptotics of solutions to those PDE problems using the results for the process $X_{t}^{\varepsilon}$ and vice versa. One can also apply the probability results to certain non-linear PDE problems related to the process. For example, certain problems for reaction-diffusion systems can be considered in this way (compare with [4], Chapters 5-7).

Finally, we note that the problem considered in this paper can be viewed as a problem concerning the long-time influence of small perturbations: the process $\tilde{X}_{t}^{\varepsilon}=X_{\varepsilon t}^{\varepsilon}$ starting at $(i, z)$ can be viewed as a small perturbation of the process $\tilde{X}_{t}$ whose first component is $\Xi_{t}^{z}$ starting at $i$ and the second component $z \in \mathbb{R}^{d}$ does not evolve in time.

A general approach to the study of the long-time influence of perturbations (see [5], [6], [7]) is to consider the projection of $X_{t}^{\varepsilon}=\tilde{X}_{t / \varepsilon}$ onto the simplex of invariant probability measures of the unperturbed process. In the case when the unperturbed process is $\tilde{X}_{t}$, the set $M_{\text {erg }}$ of the extreme points of the simplex (ergodic invariant measures) consists of the measures of the form $\mu(z) \times \delta_{z}$ (where $z \notin G$ and $\mu(z)$ is the invariant measure for $\left.\Xi_{t}^{z}\right)$ and of the measures of the form $\mu^{1}(z) \times \delta_{z}$ and $\mu^{2}(z) \times \delta_{z}$ (where $z \in G$ and $\mu^{1}(z), \mu^{2}(z)$ are invariant for $\Xi_{t}^{z}$ on $R_{1}$ and $R_{2}$, respectively). The projection of a point $(i, z), i \in\{1, \ldots, n\}, z \in \mathbb{R}^{d}$, from the phase space of $X_{t}^{\varepsilon}$ onto $M_{\mathrm{erg}}$ is $\mu(z) \times \delta_{z}$ (if $z \notin G$ ), or $\mu^{1}(z) \times \delta_{z}$ (if $z \in G, i \in R_{1}$ ), or $\mu^{2}(z) \times \delta_{z}$ (if $z \in G, i \in R_{2}$ ). Note that $M_{\mathrm{erg}}$ can be parametrized by the set of pairs $(l, z), z \in \mathbb{R}^{d}, l \in\{1,2\}$ if $z \in G$ and $l=0$ if $z \notin G$. The main result of the paper is that the projection of $X_{t}^{\varepsilon}$ onto $M_{\operatorname{erg}}$ converges to a Markov process on $M_{\mathrm{erg}}$. In the example shown in Figure 1, the set $M_{\mathrm{erg}}$ can be identified with the graph $S$.

\section{The fast-slow system}

In this section, we will introduce the fast-slow system $X_{t}^{\varepsilon}=\left(\xi_{t}^{\varepsilon}, z_{t}^{\varepsilon}\right)$. (Sometimes we will write $X_{t}^{x, \varepsilon}$ to indicate the dependence on the initial position $x$ ). The fast component $\xi_{t}^{\varepsilon}$ evolves as a Markov chain, whose transition rates depend on the slow variable. The 
Averaging in the case of multiple invariant measures for the fast system

slow component $z_{t}^{\varepsilon}$ solves an ODE or an SDE with the right hand side that depends on the fast variable.

In order to define the fast-slow system, we first introduce a family of Markov chains that, roughly speaking, governs the fast motion (and then we will take the evolution of the slow variable into account). Namely, let $q_{i j}(z) \geq 0,1 \leq i, j \leq n, i \neq j$ be a family of transition rates for a Markov chain $\Xi_{t}^{z}$ that depends on the parameter $z \in \mathbb{R}$. Each of the functions $q_{i j}(z)$ is assumed to be continuous.

We assume that $\Xi_{t}^{z}$ is ergodic for each $z<0$, while there are two ergodic classes $R_{1}=\{1, \ldots, m\}$ and $R_{2}=\{m+1, \ldots, n\}$ for $z>0$. More precisely, we assume that $q_{i j}(z)>0$ for $z<0$, while, for $z \geq 0, q_{i j}(z)>0$ if and only if $i, j \in R_{1}$ or $i, j \in R_{2}$.

Moreover, we assume that, when $z \uparrow 0$, the quantities $q_{i j}(z)-q_{i j}(0), i \neq j$, are of the same order, namely, there are positive constants $\bar{q}_{i j}$, a function $\varphi:(-\infty, 0) \rightarrow(0, \infty)$ with $\lim _{z \uparrow 0} \varphi(z)=0$ and functions $\beta_{i j}:(-\infty, 0) \rightarrow \mathbb{R}$ with $\lim _{z \uparrow 0} \beta_{i j}(z)=0$ such that

$$
q_{i j}(z)-q_{i j}(0)=\bar{q}_{i j} \varphi(z)\left(1+\beta_{i j}(z)\right), \quad z<0, \quad i \neq j .
$$

This condition is needed in order to control the transitions of the fast component between $R_{1}$ and $R_{2}$ as the slow component approaches the origin from the left. Note that, for $z \geq 0$, by the above assumptions, we simply have $q_{i j}(z)=q_{i j}(0)=0$ if $i \in R_{1}, j \in R_{2}$ or $i \in R_{2}, j \in R_{1}$, and $\lim _{z \downarrow 0} q_{i j}(z)=q_{i j}(0)>0$ if $i \in R_{1}, j \in R_{1}$ or $i \in R_{2}, j \in R_{2}$.

Let $\mu_{i}(z), 1 \leq i \leq n, z \in \mathbb{R}$, be the invariant distribution of the Markov chain $\Xi_{t}^{z}$. It is determined uniquely for $z<0$. The following lemma will be important to understand the distribution of the fast component of the process as the slow component approaches the point $z=0$ from the left.

Lemma 2.1. Under the above assumptions, there exist the limits $\pi_{i}=\lim _{z \uparrow 0} \mu_{i}(z)>0$.

Proof. The asymptotic behavior of the invariant distributions of parameter-dependent Markov chains, such as $\Xi_{t}^{z}$ with $z \uparrow 0$, was considered in [8]. (The paper [8] concerned the meta-stable distributions for the chains, and evaluating the limit of the invariant distribution was the first step). The existence of the limit for the invariant distributions was shown under the asymptotic regularity condition, which, in our case, means that for each $i \neq j$ and $k \neq l$, the following finite or infinite limit should exist:

$$
\lim _{z \uparrow 0}\left(q_{i j}(z) / q_{k l}(z)\right) \in[0, \infty] .
$$

The existence of the limits in (2.2) follows from (2.1). Moreover, from the fact that the limit in (2.2) is positive and finite for $i, l \in R_{1}$ and $j, k \in R_{2}$ (i.e., that transition rates from $R_{1}$ to $R_{2}$ are of the same order as those from $R_{2}$ to $R_{1}$ ), it easily follows that $\pi_{i}>0$ for each $i$.

Observe that the invariant distribution for $\Xi_{t}^{z}$ is not determined uniquely for $z \geq 0$ since there are two ergodic classes for the Markov chain. However, we can select the unique invariant distribution such that $\mu_{i}(z)$ are continuous functions on $\mathbb{R}$. Define

$$
\bar{\pi}_{1}=\sum_{i \in R_{1}} \pi_{i}, \quad \bar{\pi}_{2}=\sum_{i \in R_{2}} \pi_{i} .
$$

Let $v(i, z), 1 \leq i \leq n, z \in \mathbb{R}$, be Lipschitz-continuous in $z$ for each $i$. Define

$$
\begin{gathered}
\bar{v}_{0}(z)=\sum_{i=1}^{n} v(i, z) \mu_{i}(z), \quad z<0, \\
\bar{v}_{1}(z)=\frac{1}{\bar{\pi}_{1}} \sum_{i \in R_{1}} v(i, z) \mu_{i}(z), \quad \bar{v}_{2}(z)=\frac{1}{\bar{\pi}_{2}} \sum_{i \in R_{2}} v(i, z) \mu_{i}(z), \quad z \geq 0 .
\end{gathered}
$$


Averaging in the case of multiple invariant measures for the fast system

In the case when $\varkappa=0$, we assume that

$$
v(i, z)>0
$$

for each $(i, z)$. In particular, $\bar{v}_{0}(z)>0$ for $z<0$ and $\bar{v}_{1}(z), \bar{v}_{2}(z)>0$ for $z \geq 0$. This will imply that the limiting process (defined in Section 3), which behaves deterministically, except when it hits the origin, will always move from the left to the right. Assumption (2.4) could be relaxed, resulting in a slightly more general statement, but then we would not have the explicit expression describing the behavior of the limiting process at the origin (formula (3.1)). It is not difficult to see from Theorem 5.1 that the limiting process will be purely deterministic unless $\bar{v}_{1}(0), \bar{v}_{2}(0)>0$. Assumption (2.4) is not required if there is diffusion in the slow variable (case $\varkappa=1$ below).

Let us make a simplifying assumption about the behavior of the coefficients at infinity. Namely, we assume that there is $C>0$ such that $q_{i j}(z)=q_{i j}^{l}$ for $z \leq-C$ and $q_{i j}(z)=q_{i j}^{r}$ for $z \geq C$, where $q_{i j}^{l}, q_{i j}^{r}$ do not depend on $z$. Moreover, we assume that $v(i, z)=\bar{v}^{\infty}$ for some $\bar{v}^{\infty}$ for all $1 \leq i \leq n,|z| \geq C$. These assumptions can be relaxed significantly, however, this will not concern us since we would like to focus on the behavior of the process near $z=0$. The slow component $z_{t}^{\varepsilon}$ is assumed to be continuous and to satisfy

$$
d z_{t}^{\varepsilon}=v\left(\xi_{t}^{\varepsilon}, z_{t}^{\varepsilon}\right) d t+\varkappa d W_{t}
$$

on each time interval where $\xi_{t}^{\varepsilon}$ is constant. Here $\varkappa=0$ or $\varkappa=1$ (we will consider two cases resulting in two different types of the limiting behavior). The fast component, roughly speaking, evolves as the Markov chain $\Xi_{t}^{z}$ (with $z=z_{t}^{\varepsilon}$ ), sped up by the factor $1 / \varepsilon$. However, since $z$ itself evolves in time, we need a more formal definition of the process $X_{t}^{x, \varepsilon}=\left(\xi_{t}^{x, \varepsilon}, z_{t}^{x, \varepsilon}\right)$. Namely, the process starts at $x=(i, z) \in M$, and moves along the $z$-axis during a random time interval $[0, \sigma)$. For $t \in[0, \sigma), z_{t}^{x, \varepsilon}$ solves

$$
d z_{t}=v\left(i, z_{t}\right) d t+\varkappa d W_{t} .
$$

At a random time $\sigma$, the process $X_{t}^{x, \varepsilon}$ jumps to a random location $\left(j, z_{\sigma}\right)$. The distribution of $\sigma$ is determined as follows. Let $Q_{i}(z)=\sum_{j \neq i} q_{i j}(z)$ and $r(t)=\varepsilon^{-1} \int_{0}^{t} Q_{i}\left(z_{s}\right) d s$. Then $\sigma \geq 0$ is such that the distribution of $r(\sigma)$ is exponential with parameter one. Given a value of $\sigma$, the probability that $X_{t}^{x, \varepsilon}$ jumps to $\left(j, z_{\sigma}\right)$ is $q_{i j}(\sigma) / Q_{i}(\sigma)$.

Having identified the location of the process at time $\sigma$, we treat it as a new starting point, and select a new (random) time interval for the jump-free motion of the process independently of the past. The construction then continues inductively. It is clear that the process just described is a RCLL (right continuous with left limits) Markov process.

The process $X_{t}^{x, \varepsilon}=\left(\xi_{t}^{x, \varepsilon}, z_{t}^{x, \varepsilon}\right)$ could be defined, equivalently, through its generator, using the Hille-Yosida theorem. We discuss the Hille-Yosida theorem and the generator of $X_{t}^{x, \varepsilon}$ next, since, in any case, a similar construction will be used to define the limiting process when $\varkappa=1$.

Let $M$ be a separable locally compact metric space, $C_{0}(M)$ be the space of continuous functions on $M$ that tend to zero at infinity (can be made arbitrarily close to zero outside a sufficiently large compact). The space $C_{0}(M)$ is endowed with the supremum norm. Let $P(t, x, B)$ be a Markov transition function (a priori not assumed to be conservative) on $M$. For $f \in C_{0}(M)$, let

$$
\left(T_{t} f\right)(x)=\int_{\mathbb{T}^{d}} f\left(x^{\prime}\right) P\left(t, x, d x^{\prime}\right), \quad t \geq 0 .
$$

We will say that $P$ satisfies condition $C_{0}$ if $T_{t} f \in C_{0}(M)$ for each $f \in C_{0}(M)$. Recall that $P$ is said to be stochastically continuous if $\lim _{t \downarrow 0} P(t, x, U)=1$ for each open neighborhood $U$ of $x$. The Hille-Yosida Theorem can be found in various textbooks (see, e.g., [3], [11]). The statement in the form most convenient for us can be found in [10], page 365 . 
Averaging in the case of multiple invariant measures for the fast system

Theorem 2.2. [Hille-Yosida]. Suppose that a linear operator $A$ on $C_{0}(M)$ has the following properties:

(a) The domain $\mathcal{D}(A)$ is dense in $C_{0}(M)$;

(b) If $f \in \mathcal{D}(A), f\left(x_{0}\right) \geq 0$ and $f\left(x_{0}\right) \geq f(x)$ for all $x \in M$, then $A f\left(x_{0}\right) \leq 0$.

(c) For every $\psi \in C_{0}(M)$, and every $\lambda>0$, there exists a solution $f \in \mathcal{D}(A)$ of the equation $\lambda f-A f=\psi$.

Then the operator $A$ is the infinitesimal generator of a semi-group $T_{t}, t \geq 0$, on $C_{0}(M)$ that is defined by a stochastically continuous Markov transition function satisfying condition $C_{0}$. The transition function with such properties is determined uniquely.

The Hille-Yosida theorem can be applied to the space $M=\{1, \ldots, n\} \times \mathbb{R}$. Let us define the linear operator $A^{\varepsilon}$ in $C_{0}(M)$. In the case $\varkappa=0$, the domain of $A^{\varepsilon}$, denoted by $\mathcal{D}\left(A^{\varepsilon}\right)$, consists of all functions $f \in C_{0}(M)$ such that $f^{\prime}(i, \cdot) \in C_{0}(\mathbb{R})$ for each $i$. For $f \in \mathcal{D}\left(A^{\varepsilon}\right)$, we define

$$
A^{\varepsilon} f(i, z)=\frac{1}{\varepsilon}\left(\sum_{j \neq i} q_{i j}(z) f(j, z)-Q_{i}(z) f(i, z)\right)+v(i, z) f^{\prime}(i, z) .
$$

In the case when $\varkappa=1$, the domain of $A^{\varepsilon}$ consists of all functions $f \in C_{0}(M)$ such that $\frac{1}{2} f^{\prime \prime}(i, \cdot)+v(i, \cdot) f^{\prime}(i, \cdot) \in C_{0}(\mathbb{R})$ for each $i$. For $f \in \mathcal{D}\left(A^{\varepsilon}\right)$, we define

$$
A^{\varepsilon} f(i, z)=\frac{1}{\varepsilon}\left(\sum_{j \neq i} q_{i j}(z) f(j, z)-Q_{i}(z) f(i, z)\right)+\frac{1}{2} f^{\prime \prime}(i, z)+v(i, z) f^{\prime}(i, z) .
$$

In both cases, it is possible to show that the conditions of the Hille-Yosida theorem are satisfied. (We skip details since, in any case, the process was already defined constructively.) Let $P^{\varepsilon}\left(t, x, d x^{\prime}\right)$ be the corresponding Markov transition function, and $T_{t}^{\varepsilon}, t \geq 0$, be the corresponding semi-group on $C_{0}(M)$. Take a sequence of functions $f_{n} \in \mathcal{D}\left(A^{\varepsilon}\right)$ with values in $[0,1]$ with compact support such that $f_{n}(i, z)=1$ for $|z| \leq n$, $\left\|A^{\varepsilon} f_{n}\right\|_{C_{0}} \leq 1 / n$. The existence of such a sequence is easily justified once we recall that the coefficients of $A^{\varepsilon}$ are constant for sufficiently large $|z|$.

Since $A^{\varepsilon}$ is the infinitesimal generator of the semi-group $T_{t}^{\varepsilon}$, we have (see Theorem I.1 of [11]), for $f \in \mathcal{D}\left(A^{\varepsilon}\right)$,

$$
T_{t}^{\varepsilon} f-f=\int_{0}^{t} T_{s}^{\varepsilon} A^{\varepsilon} f d s .
$$

Therefore,

$$
T_{t}^{\varepsilon} f_{n}(x)-f_{n}(x)=\int_{0}^{t} T_{s}^{\varepsilon} A^{\varepsilon} f_{n}(x) d s \rightarrow 0 \text { as } n \rightarrow \infty,
$$

which implies that $T_{t}^{\varepsilon} f_{n}(x) \rightarrow 1$, and therefore $P^{\varepsilon}(t, x, \cdot)$ is a probability measure. Let $X_{t}^{x, \varepsilon}=\left(\xi_{t}^{x, \varepsilon}, z_{t}^{x, \varepsilon}\right), x=(i, z) \in M$, be the corresponding Markov family. A modification of $X_{t}^{x, \varepsilon}$ can be chosen with trajectories that are right continuous and have left limits ([10], page 348).

Rewrite (2.5) as

$$
\mathrm{E} f\left(X_{t}^{x, \varepsilon}\right)-f(x)=\mathrm{E} \int_{0}^{t}\left(A^{\varepsilon} f\right)\left(X_{s}^{x, \varepsilon}\right) d s .
$$

Since $X_{t}^{x, \varepsilon}$ is a RCLL Markov process with continuous trajectories, for each $x \in M$, the process $f\left(X_{t}^{x, \varepsilon}\right)-f(x)-\int_{0}^{t}\left(A^{\varepsilon} f\right)\left(X_{s}^{x, \varepsilon}\right) d s$ is a RCLL martingale, and, for each stopping time $\tau$ with $\mathrm{E} \tau<\infty$, we get

$$
\mathrm{E} f\left(X_{\tau}^{x, \varepsilon}\right)-f(x)=\mathrm{E} \int_{0}^{\tau}\left(A^{\varepsilon} f\right)\left(X_{s}^{x, \varepsilon}\right) d s
$$


Averaging in the case of multiple invariant measures for the fast system

Recall that we earlier defined the process $X_{t}^{x, \varepsilon}$ constructively, without referring to the Hille-Yosida theorem. It is easily verified directly that the generator of this process coincides with $A^{\varepsilon}$ on $\mathcal{D}\left(A^{\varepsilon}\right)$. The Markov transition function of the process is stochastically continuous and satisfies condition $C_{0}$. At the same time, by (2.5), the semigroup is defined uniquely by the values of the generator on a dense set, and thus the generator of the constructively defined process is $A^{\varepsilon}$ (rather than a non-trivial extension).

\section{The limiting process}

Let us describe the appropriate space and the limiting process on it for the fast-slow system $X_{t}^{x, \varepsilon}=\left(\xi_{t}^{x, \varepsilon}, z_{t}^{x, \varepsilon}\right)$. Let $I_{0}=(-\infty, 0], I_{1}=\{1\} \times[0, \infty), I_{2}=\{2\} \times[0, \infty)$. These are three half-lines, with $I_{1}$ and $I_{2}$ distinguished by a label. We will identify the ends of $I_{0}, I_{1}$, and $I_{2}$, thus obtaining a graph, denoted by $S$, with three semi-infinite edges with the common vertex, which will be denoted $O$. Each point $y=(l, z) \in S$ is determined by the label of the edge $l \in\{0,1,2\}$ and the coordinate $z$, where $z \in(-\infty, 0]$ for $l=0$ and $z \in[0, \infty)$ for $l=1,2$.

First, consider the case when there is no diffusion in the slow variable $(\varkappa=0)$. The process $Y_{t}^{y}$ starting at $y=(l, z) \in S$ will move deterministically with the variable speed $\bar{v}_{0}$ on $I_{0}, \bar{v}_{1}$ on $I_{1}$, and $\bar{v}_{2}$ on $I_{2}$. For $y \in I_{0}$, we still need to describe the behavior of $Y_{t}^{y}$ once the process reaches $O$. The behavior at $O$ is random, the process proceeds to $I_{1}$ and $I_{2}$ with probabilities

$$
p_{1}=\frac{\sum_{i \in R_{1}} \pi_{i} v_{i}}{\sum_{i \in R_{1}} \pi_{i} v_{i}+\sum_{i \in R_{2}} \pi_{i} v_{i}} \quad \text { and } \quad p_{2}=\frac{\sum_{i \in R_{2}} \pi_{i} v_{i}}{\sum_{i \in R_{1}} \pi_{i} v_{i}+\sum_{i \in R_{2}} \pi_{i} v_{i}}
$$

respectively, where $v_{i}=v(i, 0)$.

Next, consider the case with diffusion $(\varkappa=1)$. The process $Y_{t}^{y}$ is a diffusion inside each of the edges. However, a gluing condition is needed to describe the behavior of the process once it reaches the vertex. Thus, it is most convenient to define the process via its generator. The domain of $A$, denoted by $\mathcal{D}(A)$, consists of all functions $f \in C_{0}(S)$ such that:

(a) $\frac{1}{2} f^{\prime \prime}(l, \cdot)+\bar{v}_{l}(\cdot) f^{\prime}(l, \cdot) \in C_{0}(S)$, i.e., the differential operator can be applied to $f$ inside each of the edges, and the resulting function can be extended to the vertex $O$, so that it becomes an element of $C_{0}(S)$.

(b) There are one-sided derivatives $f^{\prime}(l, 0)$ and

$$
f^{\prime}(0,0)=\bar{\pi}_{1} f^{\prime}(1,0)+\bar{\pi}_{2} f^{\prime}(2,0) .
$$

It is not difficult to verify that the conditions of the Hille-Yosida theorem are satisfied and that the resulting Markov transition function, denoted by $P(t, x, B)$, is a probability measure, as a function of $B$. Let $Y_{t}^{y}, y \in S$, be the corresponding Markov family and $T_{t}$ be the corresponding semigroup. In order to show that a modification with continuous trajectories exists, it is enough to check that $\lim _{t \downarrow 0} P(t, x, B) / t=0$ for each closed set $B$ that doesn't contain $x$ (Theorem I.5 of [11], see also [1]). Let $f \in \mathcal{D}(A)$ be a non-negative function that is equal to one on $B$ and whose support doesn't contain $x$. Then

$$
\lim _{t \downarrow 0} \frac{P(t, x, B)}{t} \leq \lim _{t \downarrow 0} \frac{\left(T_{t} f\right)(x)-f(x)}{t}=A f(x)=0,
$$

as required. Thus $Y_{t}^{y}$ can be assumed to have continuous trajectories.

\section{A lemma on convergence of processes}

The next lemma can be used to show convergence of families of parameter-dependent processes. We formulate it in a general setting. Consider a metric space $M$ and a Markov 
Averaging in the case of multiple invariant measures for the fast system

family $X_{t}^{x, \varepsilon}, x \in M$, of processes that depend on a parameter $\varepsilon>0$. We also consider a continuous mapping $h: M \rightarrow S$ from $M$ to a locally compact separable metric space $S$ and define the processes $Y_{t}^{x, \varepsilon}=h\left(X_{t}^{x, \varepsilon}\right), x \in M, \varepsilon>0$.

The motivation to introduce the latter family of processes comes from our desire to study the limiting behavior of $X_{t}^{x, \varepsilon}$, as $\varepsilon \downarrow 0$. However, the space $M$ is too large for our purposes, i.e., the natural state space for the limiting process consists of equivalence classes in $M$ rather than of individual points. Thus, $Y_{t}^{x, \varepsilon}$ will capture reduced dynamics, where meaningful limiting behavior can be observed.

Note that while convergence to Markov processes on $S$ as $\varepsilon \downarrow 0$ will be established, the processes $Y_{t}^{x, \varepsilon}$ need not be Markov for fixed $\varepsilon>0$. The main point of the lemma is that, in order to demonstrate the convergence of $Y_{t}^{x, \varepsilon}$ to a limiting process, it is sufficient to check that for small $\varepsilon$ the processes nearly satisfy the relation (4.1), which is similar to the martingale problem but with the ordinary expectation rather than the conditional expectation.

Lemma 4.1. Let $h: M \rightarrow S$ be a continuous mapping from a metric space $M$ to a locally compact separable metric space $S$. Let $X_{t}^{x, \varepsilon}, x \in M$, be a Markov family on $M$ that depends on a parameter $\varepsilon>0$. Suppose that the processes $Y_{t}^{x, \varepsilon}=h\left(X_{t}^{x, \varepsilon}\right)$, $x \in M, \varepsilon>0$, have continuous trajectories. Let $Y_{t}^{y}, y \in S$, be a Markov family on $S$ with continuous trajectories whose semigroup $T_{t}, t \geq 0$, preserves the space $C_{0}(S)$. (This, together with the continuity of trajectories, implies that $T_{t}$ is a Feller semi-group, i.e., $T_{t} f$, viewed as a function of $t$, is a right-continuous from $[0, \infty)$ to $C_{0}(S)$ for each $f$.) Let $A: \mathcal{D}(A) \rightarrow C_{0}(S)$ denote the infinitesimal generator of this family, where $\mathcal{D}(A)$ is the domain of the generator. Let $\Psi$ be a dense linear subspace of $C_{0}(S)$ and $\mathcal{D}$ be a linear subspace of $\mathcal{D}(A)$, and suppose that $\Psi$ and $\mathcal{D}$ have the following properties:

(1) There is $\lambda>0$ such that for each $f \in \Psi$ the equation $\lambda F-A F=f$ has a solution $F \in \mathcal{D}$.

(2) For each $T>0$, each $f \in \mathcal{D}$, and each compact $K \subseteq S$,

$$
\lim _{\varepsilon \downarrow 0} \mathrm{E}\left(f\left(Y_{T}^{x, \varepsilon}\right)-f\left(Y_{0}^{x, \varepsilon}\right)-\int_{0}^{T} A f\left(Y_{t}^{x, \varepsilon}\right) d t\right)=0,
$$

uniformly in $x \in h^{-1}(K)$. Suppose that the family of measures on $C([0, \infty), S)$ induced by the processes $Y_{t}^{x, \varepsilon}, \varepsilon>0$, is tight for each $x \in M$.

Then, for each $x \in M$, the measures induced by the processes $Y_{t}^{x, \varepsilon}$ converge weakly, as $\varepsilon \downarrow 0$, to the measure induced by the process $Y_{t}^{h(x)}$.

Proof. Fix $x \in M$. Since the family of measures on $C([0, \infty), S)$ induced by the processes $Y_{t}^{x, \varepsilon}, \varepsilon>0$, is tight, we can find a process $Z_{t}^{x}$ with continuous trajectories and a sequence $\varepsilon_{n} \downarrow 0$ such that $Y_{t}^{x, \varepsilon_{n}}$ converge to $Z_{t}^{x}$ in distribution as $n \rightarrow \infty$. The desired result will immediately follow if we demonstrate that the distribution of $Z_{t}^{x}$ coincides with the distribution of $Y_{t}^{h(x)}$ (and thus does not depend on the choice of the sequence $\varepsilon_{n}$ ). We will show that $Z_{t}^{x}$ is a solution of the martingale problem for $\left(\left.A\right|_{\mathcal{D}}, h(x)\right)$, i.e., for each $T_{2}>T_{1} \geq 0$ and $f \in \mathcal{D}$,

$$
\mathrm{E}\left(f\left(Z_{T_{2}}^{x}\right)-f\left(Z_{T_{1}}^{x}\right)-\int_{T_{1}}^{T_{2}} A f\left(Z_{t}^{x}\right) d t \mid \mathcal{F}_{T_{1}}^{Z^{x}}\right)=0, \quad Z_{0}^{x}=h(x) .
$$

First, however, let us discuss the uniqueness for solutions of the martingale problem. We claim that:

(a) $\mathcal{D}$ is dense in $C_{0}(S)$.

(b) Range $\left(\lambda-\left.A\right|_{\mathcal{D}}\right)$ is dense in $C_{0}(S)$.

(c) For each pair of measures $\mu_{1}, \mu_{2}$ on $S$, the equality $\int_{S} f d \mu_{1}=\int_{S} f d \mu_{2}$ for all $f \in C_{0}(S)$ implies that $\mu_{1}=\mu_{2}$. 
To demonstrate (a), take an arbitrary $\delta>0$ and $F_{0} \in \mathcal{D}(A)$. Let $g_{0}=\lambda F_{0}-A F_{0}$, and take $g^{\prime} \in \Psi$ such that $\left\|g^{\prime}-g_{0}\right\| \leq \lambda \delta$. Let $F^{\prime} \in \mathcal{D}$ be such that $\lambda F^{\prime}-A F^{\prime}=g^{\prime}$. Then, since $A$ is the generator of a strongly continuous semigroup on $C_{0}(S)$, from the Hille-Yosida theorem it follows that $\left\|F^{\prime}-F_{0}\right\| \leq\left\|g^{\prime}-g_{0}\right\| / \lambda \leq \delta$. This implies (a) since $\mathcal{D}(A)$ is dense in $C_{0}(S)$. Note that (b) follows from the existence of a solution $F \in \mathcal{D}$ to $\lambda F-A F=f \in \Psi$ and the density of $\Psi$, while (c) is obvious. The validity of (a)-(c) is enough to conclude that the distribution on $C([0, \infty), S)$ of a process with continuous paths satisfying (4.2) is uniquely determined (Theorem 4.1, Chapter 4 in [2]).

Note that (4.2) is satisfied if $Z_{t}^{x}$ is replaced by $Y_{t}^{h(x)}$ since $\mathcal{D} \subseteq \mathcal{D}(A)$ and $A$ the the generator of the family $Y_{t}^{y}, y \in S$. Therefore, $Z_{t}^{x}$ and $Y_{t}^{h(x)}$ have the same distribution if (4.2) holds. It remains to prove (4.2).

Note that $Z_{t}^{x}$ is a solution of the martingale problem for $\left(\left.A\right|_{\mathcal{D}}, h(x)\right)$ if and only if

$$
\mathrm{E}\left(\left(\prod_{i=1}^{k} g_{i}\left(Z_{t_{i}}^{x}\right)\right)\left(f\left(Z_{T_{2}}^{x}\right)-f\left(Z_{T_{1}}^{x}\right)-\int_{T_{1}}^{T_{2}} A f\left(Z_{t}^{x}\right) d t\right)\right)=0, \quad Z_{0}^{x}=h(x),
$$

whenever $f \in \mathcal{D}, 0 \leq t_{1}<\ldots<t_{k} \leq T_{1}$, and $g_{1}, \ldots, g_{k} \in C_{0}(S)$. Since $Y_{t}^{x, \varepsilon_{n}}=h\left(X_{t}^{x, \varepsilon_{n}}\right)$ converge to $Z_{t}^{x}$ in distribution, we have

$$
\begin{gathered}
\mathrm{E}\left(\left(\prod_{i=1}^{k} g_{i}\left(Z_{t_{i}}^{x}\right)\right)\left(f\left(Z_{T_{2}}^{x}\right)-f\left(Z_{T_{1}}^{x}\right)-\int_{T_{1}}^{T_{2}} A f\left(Z_{t}^{x}\right) d t\right)\right)= \\
\lim _{n \rightarrow \infty} \mathrm{E}\left(\left(\prod_{i=1}^{k} g_{i}\left(h\left(X_{t_{i}}^{x, \varepsilon_{n}}\right)\right)\right)\left(f\left(h\left(X_{T_{2}}^{x, \varepsilon_{n}}\right)\right)-f\left(h\left(X_{T_{1}}^{x, \varepsilon_{n}}\right)\right)-\int_{T_{1}}^{T_{2}} A f\left(h\left(X_{t}^{x, \varepsilon_{n}}\right)\right) d t\right)\right)= \\
\lim _{n \rightarrow \infty} \mathrm{E}\left(\left(\prod_{i=1}^{k} g_{i}\left(h\left(X_{t_{i}}^{x, \varepsilon_{n}}\right)\right)\right) \mathrm{E}\left(f\left(h\left(X_{T_{2}}^{x, \varepsilon_{n}}\right)\right)-f\left(h\left(X_{T_{1}}^{x, \varepsilon_{n}}\right)\right)-\int_{T_{1}}^{T_{2}} A f\left(h\left(X_{t}^{x, \varepsilon_{n}}\right)\right) d t \mid \mathcal{F}_{T_{1}}^{X^{x, \varepsilon_{n}}}\right)\right) .
\end{gathered}
$$

By the Markov property of the family $X_{t}^{x, \varepsilon_{n}}$,

$$
\begin{gathered}
\mathrm{E}\left(f\left(h\left(X_{T_{2}}^{x, \varepsilon_{n}}\right)\right)-f\left(h\left(X_{T_{1}}^{x, \varepsilon_{n}}\right)\right)-\int_{T_{1}}^{T_{2}} A f\left(h\left(X_{t}^{x, \varepsilon_{n}}\right)\right) d t \mid \mathcal{F}_{T_{1}}^{X^{x, \varepsilon_{n}}}\right)= \\
\left.\mathrm{E}\left(f\left(h\left(X_{T_{2}-T_{1}}^{x^{\prime}, \varepsilon_{n}}\right)\right)-f\left(h\left(X_{0}^{x^{\prime}, \varepsilon_{n}}\right)\right)-\int_{0}^{T_{2}-T_{1}} A f\left(h\left(X_{t}^{x^{\prime}, \varepsilon_{n}}\right)\right) d t\right)\right|_{x^{\prime}=X_{T_{1}}^{x, \varepsilon_{n}}},
\end{gathered}
$$

which tends to zero in distribution, as follows from (4.1) and from the tightness of the sequence of random variables $X_{T_{1}}^{x, \varepsilon_{n}}$. Therefore, using the boundedness of $f, A f$, and $g_{1}, \ldots, g_{k}$, we conclude that

$$
\mathrm{E}\left(\left(\prod_{i=1}^{k} g_{i}\left(Z_{t_{i}}^{x}\right)\right)\left(f\left(Z_{T_{2}}^{x}\right)-f\left(Z_{T_{1}}^{x}\right)-\int_{T_{1}}^{T_{2}} A f\left(Z_{t}^{x}\right) d t\right)\right)=0 .
$$

Finally, $Z_{0}^{x}=h(x)$ since $Y_{0}^{x, \varepsilon_{n}}=h\left(X_{0}^{x, \varepsilon_{n}}\right)=h(x)$ for all $n$.

\section{Convergence of the fast-slow process}

\subsection{The case with no diffusion}

Consider first a simplified version of the problem: assume that the fast-slow system $X_{t}^{x, \varepsilon}=\left(\xi_{t}^{x, \varepsilon}, z_{t}^{x, \varepsilon}\right)$ is defined as in Section 2, but $q_{i j}(z)>0$ for $i \neq j$ (and thus $\Xi_{t}^{z}$ is ergodic) for each $z \in \mathbb{R}$. In this case, the fast Markov chain has a unique invariant distribution, which will be denoted by $\mu_{i}(z), 1 \leq i \leq n$, for each $z \in \mathbb{R}$. Define $Y_{t}^{y}, y \in \mathbb{R}$, to be the deterministic motion on the real line with the velocity $\bar{v}(y)=\sum_{i=1}^{n} v(i, z) \mu_{i}(z)$, 
Averaging in the case of multiple invariant measures for the fast system

$z \in \mathbb{R}$. The domain $\mathcal{D}(A)$ of its generator $A$ consists of all functions $f \in C_{0}(\mathbb{R})$ such that $f^{\prime} \in C_{0}(\mathbb{R})$, while $A f(y)=\bar{v}(y) f^{\prime}(y)$. Let $h: M \rightarrow \mathbb{R}$ be the projection $h(i, z)=z$. The following lemma is a standard averaging result. It can be used to describe the behavior of the fast-slow system when the slow component is outside of an arbitrarily small neighborhood of the origin.

Lemma 5.1. Suppose that $q_{i j}>0$ for $i \neq j, z \in \mathbb{R}$. For each $x \in M$, the measures induced by the processes $Y_{t}^{x, \varepsilon}=h\left(X_{t}^{x, \varepsilon}\right)$ on $\mathbb{R}$ converge weakly, as $\varepsilon \downarrow 0$, to the measure induced by the process $Y_{t}^{h(x)}$.

Proof. We apply Lemma 4.1 with $S=\mathbb{R}, \Psi=\mathcal{D}=\mathcal{D}(A)$. Thus we need to justify (4.1) for $f \in \mathcal{D}(A)$. Define $\tilde{f}(i, z)=f(z), 1 \leq i \leq n$. Using (2.6) (which is still valid in this simplified case) applied to $\tilde{f}$ with $\tau=T$, we can write

$$
\begin{gathered}
\mathrm{E}\left(f\left(Y_{T}^{x, \varepsilon}\right)-f\left(Y_{0}^{x, \varepsilon}\right)-\int_{0}^{T} A f\left(Y_{t}^{x, \varepsilon}\right) d t\right)= \\
\mathrm{E}\left(f\left(Y_{T}^{x, \varepsilon}\right)-f\left(Y_{0}^{x, \varepsilon}\right)-\int_{0}^{T} A f\left(Y_{t}^{x, \varepsilon}\right) d t\right)-\mathrm{E}\left(\tilde{f}\left(X_{T}^{x, \varepsilon}\right)-\tilde{f}(x)-\int_{0}^{T} A^{\varepsilon} \tilde{f}\left(X_{t}^{x, \varepsilon}\right) d t\right)= \\
\mathrm{E} \int_{0}^{T}\left(A^{\varepsilon} \tilde{f}\left(X_{t}^{x, \varepsilon}\right)-A f\left(Y_{t}^{x, \varepsilon}\right)\right) d t=\mathrm{E} \int_{0}^{T}\left(v\left(X_{t}^{x, \varepsilon}\right)-\bar{v}\left(z_{t}^{x, \varepsilon}\right)\right) f^{\prime}\left(z_{t}^{x, \varepsilon}\right) d t .
\end{gathered}
$$

It easily follows from the explicit construction of $X_{t}^{x, \varepsilon}$ (Section 2) that the expression in the right hand side tends to zero uniformly in $x$.

Now let us consider the original situation with two ergodic classes for the Markov chain when $z \geq 0$. Recall that $S$ is now a graph with three semi-infinite edges, $I_{0}, I_{1}$, and $I_{2}$, with the common vertex $O$. The process $Y_{t}^{y}$ on $S$ has been defined in Section 3 (the case $\varkappa=0$ ). The motion is deterministic on each of the edges, while the behavior at $O$ is random - the process proceeds to $I_{1}$ or $I_{2}$ with the prescribed probabilities $p_{1}$ and $p_{2}$, respectively.

Let $h$ be the mapping of $M=\{1, \ldots, n\} \times \mathbb{R}$ to $S$ defined as follows:

$$
h(i, z)= \begin{cases}(0, z), & z \leq 0, \\ (1, z), & i \in R_{1}, z \geq 0, \\ (2, z), & i \in R_{2}, z \geq 0 .\end{cases}
$$

Theorem 5.2. Suppose that $\varkappa=0$ and that the assumptions made in Section 2 are satisfied (in particular, the Markov chain $\Xi_{t}^{z}$ has two ergodic classes for each $z \geq 0$ ). For each $x \in M$, the measures induced by the processes $Y_{t}^{x, \varepsilon}=h\left(X_{t}^{x, \varepsilon}\right)$ on $S$ converge weakly, as $\varepsilon \downarrow 0$, to the measure induced by the process $Y_{t}^{h(x)}$.

Proof. Lemma 4.1 is not directly applicable now because the semigroup that corresponds to the process $Y_{t}^{y}$ does not preserve $C_{0}(S)$. However, outside of an arbitrarily small neighborhood of the set $h^{-1}(O)$, the limiting motion of $Y_{t}^{x, \varepsilon}$ is given by $Y_{t}^{h(x)}$, as follows from Lemma 5.1. To complete the proof, we need to show that if $X_{t}^{x, \varepsilon}$ starts slightly to the left of $h^{-1}(O)$, then it quickly moves to the right of $h^{-1}(O)$ and $\xi_{t}^{x, \varepsilon}$ ends up in the first ergodic class with probability close to $p_{1}$ (defined in (3.1)).

More precisely, let

$$
\tau_{\delta}^{x, \varepsilon}=\inf \left\{t \geq 0: z_{t}^{x, \varepsilon}=\delta\right\} .
$$

It is sufficient to show that for each $\eta>0$ there is $\delta_{0}>0$ such that each $\delta \in\left(0, \delta_{0}\right]$ there is $\varepsilon_{0}>0$ such that for $\varepsilon \in\left(0, \varepsilon_{0}\right]$, we have

$$
\begin{gathered}
\mathrm{E} \tau_{\delta}^{x, \varepsilon}<\eta, \\
\left|\mathrm{P}\left(\xi_{\tau_{\delta}^{x, \varepsilon}}^{x, \varepsilon} \in R_{1}\right)-p_{1}\right|<\eta,
\end{gathered}
$$


whenever $x=(i,-\delta)$. From the explicit construction of $X_{t}^{x, \varepsilon}$ (Section 2), it is clear that $z_{t}^{x, \varepsilon}$ increases, while on $[-\delta, \delta]$, with the speed that is bounded from below by $\inf _{i, z \in[-\delta, \delta]} v(i, z)>0$. This implies (5.2). To prove (5.3), we define $f_{\varepsilon}(i, z), z \in[-\delta, \delta]$, as the solution of the system of ODEs

$$
\frac{d f_{\varepsilon}(i, z)}{d z}=\frac{(v(i, z))^{-1}}{\varepsilon}\left(Q_{i}(z) f_{\varepsilon}(i, z)-\sum_{j \neq i} q_{i j}(z) f_{\varepsilon}(j, z)\right)
$$

with the terminal condition

$$
f_{\varepsilon}(i, \delta)=\mathbf{f}_{i}:= \begin{cases}1, & i \in R_{1} \\ 0, & i \in R_{2} .\end{cases}
$$

We extend $f_{\varepsilon}$ to be defined on $M$ so that $f_{\varepsilon} \in \mathcal{D}\left(A^{\varepsilon}\right)$. Observe that, by construction, $A^{\varepsilon} f_{\varepsilon}(i, z)=0$ when $z \in[-\delta, \delta]$. Therefore, applying (2.6) with $\tau=\tau_{\delta}^{x, \varepsilon}$ and $x=(i,-\delta)$, we obtain

$$
\mathrm{P}\left(\xi_{\tau_{\delta}^{x, \varepsilon}}^{x, \varepsilon} \in R_{1}\right)=f_{\varepsilon}(i,-\delta) .
$$

Thus it remains to analyze the asymptotics of the solution to the ODE. Let $N(z)$ be the matrix, whose diagonal elements are $N_{i i}(z)=-(v(i, z))^{-1} Q_{i}(z)$ and off-diagonal elements are $N_{i j}(z)=(v(i, z))^{-1} q_{i j}(z)$. Let

$$
N^{\delta}=\frac{1}{2 \delta} \int_{-\delta}^{\delta} N(z) d z
$$

Solving the linear ODE, we get

$$
f_{\varepsilon}(\cdot,-\delta)=\exp \left(\frac{2 \delta}{\varepsilon} N^{\delta}\right) \mathbf{f} .
$$

When $\delta$ is small, $N^{\delta}$ is a small perturbation of the matrix $N(0)$. Namely, let

$$
H^{\delta}=N^{\delta}-N(0) \text {. }
$$

All the entries of $H^{\delta}$ tend to zero when $\delta \downarrow 0$. Observe that all the off-diagonal entries of $N^{\delta}$ are positive for each $\delta$, and the sum of elements in each row is equal to zero. Therefore, zero is the simple eigenvalue of $N^{\delta}$ with the right eigenvector equal to $\mathbf{e}=(1, \ldots, 1)^{T}$, the real parts of the other eigenvalues are negative.

Let $\Pi_{\mathbf{e}}^{\delta}(\mathbf{f})$ be the projection of $\mathbf{f}$ onto e along the space spanned by the remaining eigenvectors (and generalized eigenvectors) of the matrix $N^{\delta}$. Then

$$
\lim _{\varepsilon \downarrow 0} f_{\varepsilon}(i,-\delta)=\left(\Pi_{\mathbf{e}}^{\delta}(\mathbf{f})\right)_{i}
$$

for each $i$, and it remains to show that $\left(\Pi_{e}^{\delta}(\mathbf{f})\right)_{i}$ (which does not depend on $i$ ) is close to $p_{1}$ for small $\delta$.

Observe that zero is the top eigenvalue of $N(0)$ with two linearly independent right eigenvectors $e$ and $f$ and two linearly independent left eigenvectors:

$$
\begin{aligned}
& \mathbf{w}_{i}^{1}= \begin{cases}\pi_{i} v_{i}, & i \in R_{1}, \\
0, & i \in R_{2},\end{cases} \\
& \mathbf{w}_{i}^{2}= \begin{cases}0, & i \in R_{1}, \\
\pi_{i} v_{i}, & i \in R_{2},\end{cases}
\end{aligned}
$$

where $v_{i}=v(i, 0)$ and the quantities $\pi_{i}$ were introduced in Lemma 2.1. Let $\lambda_{1}^{\delta}$ be the eigenvalue of $N^{\delta}$ with the second-largest real part (the top eigenvalue is zero). It is determined uniquely for small $\delta$. Let $\mathbf{g}^{\delta}$ be the corresponding right eigenvector (determined up to a constant factor). 
Averaging in the case of multiple invariant measures for the fast system

Lemma 5.3. The vector $\mathbf{g}^{\delta}$ can be represented as

$$
\mathbf{g}^{\delta}=\mathbf{e}+\alpha^{\delta} \mathbf{f}+\mathbf{r}^{\delta},
$$

where $\mathbf{r}^{\delta}$ belongs to the space spanned by the eigenvectors (and generalized eigenvectors) of $N(0)$, other than e and $\mathbf{f}$. The coefficient $\alpha^{\delta}$ is bounded away from zero, and $\mathbf{r}^{\delta}$ tends to zero when $\delta \downarrow 0$.

Proof. Recall that the right eigenspace corresponding to the top eigenvalue (equal to zero) of the matrix $N(0)$ is two-dimensional (spanned by e and $\mathbf{f}$ ). For $\delta>0$, the perturbed matrix has one top eigenvalue (equal to zero) and another that is close to zero. The space spanned by the corresponding eigenvectors is close to the space spanned by e and $\mathbf{f}$. Therefore, $\mathbf{g}^{\delta}$ can be represented as a linear combination of $\mathbf{e}, \mathbf{f}$, and a correction term that is much smaller than the norm of $\mathrm{g}^{\delta}$. We only need to show that the coefficients at $\mathbf{e}$ and $\mathbf{f}$ in this representation are of the same order when $\delta$ is small. The statements about the asymptotic behavior of the eigenvectors used below easily follow from the block-diagonal structure of the matrix $N(0)$.

Let $i_{1}(\delta)$ be such that $\left|\mathbf{g}_{i_{1}(\delta)}^{\delta}\right|=\max _{1 \leq i \leq n}\left|\mathbf{g}_{i}^{\delta}\right|$. Assume, for now, that $i_{1}(\delta) \in R_{1}$ for all sufficiently small $\delta$. Then, since $N^{\delta}$ is a small perturbation of $N(0)$ and $\lambda_{1}^{\delta} \rightarrow 0$ as $\delta \downarrow 0$, $N^{\delta} \mathbf{g}^{\delta}=\lambda_{1}^{\delta} \mathbf{g}^{\delta}$ easily implies that $\mathbf{g}_{i}^{\delta} / \mathbf{g}_{i_{1}(\delta)}^{\delta} \rightarrow 1$ as $\delta \downarrow 0$ for all $i \in R_{1}$.

Let $\tilde{\mathbf{w}}^{\delta}$ be the normalized left eigenvector for $N^{\delta}$ with eigenvalue zero. From $\tilde{\mathbf{w}}^{\delta} N^{\delta}=$ 0 and $N^{\delta} \mathbf{g}^{\delta}=\lambda_{1}^{\delta} \mathbf{g}^{\delta}$ it follows that $\left\langle\tilde{\mathbf{w}}^{\delta}, \mathbf{g}^{\delta}\right\rangle=0$. Let $i_{2}(\delta)$ be such that $\mathbf{g}_{i_{2}(\delta)}^{\delta}=\max _{i \in R_{2}}\left|\mathbf{g}_{i}^{\delta}\right|$. Observe that $\tilde{\mathbf{w}}_{i}^{\delta} \rightarrow \mathbf{w}_{i}^{1}$ for $i \in R_{1}$, and $\tilde{\mathbf{w}}_{i}^{\delta} \rightarrow \mathbf{w}_{i}^{2}$ for $i \in R_{2}$. Therefore,

$$
c_{1}\left|\mathbf{g}_{i_{1}(\delta)}^{\delta}\right| \leq\left|\mathbf{g}_{i_{2}}^{\delta}\right| \leq c_{2}\left|\mathbf{g}_{i_{1}(\delta)}^{\delta}\right|
$$

for some positive constants $c_{1}$ and $c_{2}$. As above, $\mathbf{g}_{i}^{\delta} / \mathbf{g}_{i_{2}(\delta)}^{\delta} \rightarrow 1$ as $\delta \downarrow 0$ for all $i \in R_{2}$. From the facts that $\left\langle\tilde{\mathbf{w}}^{\delta}, \mathbf{g}^{\delta}\right\rangle=0, \tilde{\mathbf{w}}_{i}^{\delta} \rightarrow \mathbf{w}_{i}^{1}$ for $i \in R_{1}$, and $\tilde{\mathbf{w}}_{i}^{\delta} \rightarrow \mathbf{w}_{i}^{2}$ for $i \in R_{2}$, it follows that $\mathbf{g}_{i}^{\delta}, i \in R_{1}$, are of the opposite sign from $\mathbf{g}_{i}^{\delta}, i \in R_{2}$.

The vector $\mathbf{g}^{\delta}$ can be represented as a sum of three components, $\mathbf{g}^{\delta}=\mathbf{a}^{\delta}+\mathbf{b}^{\delta}+\mathbf{c}^{\delta}$, where $\mathbf{a}^{\delta}$ is a multiple of $\mathbf{e}, \mathbf{b}^{\delta}$ is a multiple of $\mathbf{f}$, and $\mathbf{c}^{\delta}$ is in the space spanned by the eigenvectors (and generalized eigenvectors) of $N(0)$, other than e and f. Observe that $\left\|\mathbf{c}^{\delta}\right\| /\left\|\mathbf{g}^{\delta}\right\| \rightarrow 0$ as $\delta \downarrow 0$ since $\mathbf{e}$ and $\mathbf{f}$ span the eigenspace corresponding to the top eigenvalue of $N(0)$ and $\mathbf{g}^{\delta}$ belongs to a small perturbation of that space. Moreover, from (5.6) and the fact that $\mathbf{g}_{i}^{\delta}, i \in R_{1}$, and $\mathbf{g}_{i}^{\delta}, i \in R_{2}$, are of the opposite sign, it follows that $\left\|\mathbf{a}^{\delta}\right\| /\left\|\mathbf{b}^{\delta}\right\|$ is bounded from above and below. Therefore, (5.5) is possible with $\alpha^{\delta}$ bounded away from zero and infinity.

Finally, it remains to note that our condition $i_{1}(\delta) \in R_{1}$ does not lead to any loss of generality.

Since $\mathrm{g}^{\delta}$ is the eigenvector of $N^{\delta}$, we get

$$
\left(N(0)+H^{\delta}\right)\left(\mathbf{e}+\alpha^{\delta} \mathbf{f}+\mathbf{r}^{\delta}\right)=\lambda_{1}^{\delta}\left(\mathbf{e}+\alpha^{\delta} \mathbf{f}+\mathbf{r}^{\delta}\right) .
$$

Taking the scalar product with $\mathbf{w}^{1}$ and $\mathbf{w}^{2}$ on both sides and noting that $H^{\delta} \mathbf{e}=0$, we obtain

$$
\begin{aligned}
& \alpha^{\delta}\left\langle\mathbf{w}^{1}, H^{\delta} \mathbf{f}\right\rangle+\left\langle\mathbf{w}^{1}, H^{\delta} \mathbf{r}^{\delta}\right\rangle=\lambda_{1}^{\delta}\left\langle\mathbf{w}^{1}, \mathbf{e}+\alpha^{\delta} \mathbf{f}\right\rangle, \\
& \alpha^{\delta}\left\langle\mathbf{w}^{2}, H^{\delta} \mathbf{f}\right\rangle+\left\langle\mathbf{w}^{2}, H^{\delta} \mathbf{r}^{\delta}\right\rangle=\lambda_{1}^{\delta}\left\langle\mathbf{w}^{2}, \mathbf{e}+\alpha^{\delta} \mathbf{f}\right\rangle .
\end{aligned}
$$

Therefore,

$$
\left(\alpha^{\delta}\left\langle\mathbf{w}^{1}, H^{\delta} \mathbf{f}\right\rangle+\left\langle\mathbf{w}^{1}, H^{\delta} \mathbf{r}^{\delta}\right\rangle\right)\left\langle\mathbf{w}^{2}, \mathbf{e}+\alpha^{\delta} \mathbf{f}\right\rangle=\left(\alpha^{\delta}\left\langle\mathbf{w}^{2}, H^{\delta} \mathbf{f}\right\rangle+\left\langle\mathbf{w}^{2}, H^{\delta} \mathbf{r}^{\delta}\right\rangle\right)\left\langle\mathbf{w}^{1}, \mathbf{e}+\alpha^{\delta} \mathbf{f}\right\rangle .
$$


Averaging in the case of multiple invariant measures for the fast system

Observe that

$$
\left\langle\mathbf{w}^{1}, H^{\delta} \mathbf{r}^{\delta}\right\rangle=o\left(\alpha^{\delta}\left\langle\mathbf{w}^{1}, H^{\delta} \mathbf{f}\right\rangle\right), \quad\left\langle\mathbf{w}^{2}, H^{\delta} \mathbf{r}^{\delta}\right\rangle=o\left(\alpha^{\delta}\left\langle\mathbf{w}^{2}, H^{\delta} \mathbf{f}\right\rangle\right), \quad \text { as } \delta \downarrow 0,
$$

and therefore,

$$
\lim _{\delta \downarrow 0} \frac{\left\langle\mathbf{w}^{1}, H^{\delta} \mathbf{f}\right\rangle\left\langle\mathbf{w}^{2}, \mathbf{e}+\alpha^{\delta} \mathbf{f}\right\rangle}{\left\langle\mathbf{w}^{2}, H^{\delta} \mathbf{f}\right\rangle\left\langle\mathbf{w}^{1}, \mathbf{e}+\alpha^{\delta} \mathbf{f}\right\rangle}=1 .
$$

Solving for $\alpha^{\delta}$ (using the definition of $H^{\delta}$ from (5.4) and of $\bar{q}_{i j}$ from (2.1)), we get

$$
\lim _{\delta \downarrow 0} \alpha^{\delta}=-1-\frac{\left(\sum_{i \in R_{1}} \sum_{j \in R_{2}} \bar{q}_{i j} \pi_{i}\right)\left(\sum_{i \in R_{2}} \mathbf{w}_{i}^{2}\right)}{\left(\sum_{i \in R_{2}} \sum_{j \in R_{1}} \bar{q}_{i j} \pi_{i}\right)\left(\sum_{i \in R_{1}} \mathbf{w}_{i}^{1}\right)}=-1-\frac{\sum_{i \in R_{2}} \mathbf{w}_{i}^{2}}{\sum_{i \in R_{1}} \mathbf{w}_{i}^{1}} .
$$

From (5.5), it follows that

$$
\lim _{\delta \downarrow 0}\left(\Pi_{\mathbf{e}}^{\delta}(\mathbf{f})\right)_{i}=-1 / \lim _{\delta \downarrow 0} \alpha^{\delta}=\frac{\sum_{i \in R_{1}} \mathbf{w}_{i}^{1}}{\sum_{i \in R_{1}} \mathbf{w}_{i}^{1}+\sum_{i \in R_{2}} \mathbf{w}_{i}^{2}}=p_{1},
$$

as required (see the definition of $p_{1}$ from (3.1)).

\subsection{The case with diffusion}

Now we consider the fast-slow system $X_{t}^{x, \varepsilon}=\left(\xi_{t}^{x, \varepsilon}, z_{t}^{x, \varepsilon}\right)$ defined in Section 2, with $\varkappa=1$. The filtration generated by the process will be denoted by $\mathcal{F}_{t}^{x, \varepsilon}$. The process $Y_{t}^{y}$ on the graph $S$ is now a diffusion (defined in Section 3 via its generator). The mapping $h$ is the same as in (5.1).

Theorem 5.4. Suppose that $\varkappa=1$ and that the assumptions made in Section 2 are satisfied (in particular, the Markov chain $\Xi_{t}^{z}$ has two ergodic classes for each $z \geq 0$ ). For each $x \in M$, the measures induced by the processes $Y_{t}^{x, \varepsilon}=h\left(X_{t}^{x, \varepsilon}\right)$ on $S$ converge weakly, as $\varepsilon \downarrow 0$, to the measure induced by the process $Y_{t}^{h(x)}$.

Proof. Let $T>0, f \in \mathcal{D}(A)$, and let $K$ be a compact subset of $S$. It is clear that the family of measures on $C([0, \infty), S)$ induced by the processes $Y_{t}^{x, \varepsilon}, \varepsilon>0$, is tight for each $x \in M$. Thus, by Lemma 4.1 , it is sufficient to prove that, given $\eta>0$, we have

$$
\left|\mathrm{E}\left(f\left(Y_{T}^{x, \varepsilon}\right)-f\left(Y_{0}^{x, \varepsilon}\right)-\int_{0}^{T} A f\left(Y_{t}^{x, \varepsilon}\right) d t\right)\right| \leq \eta,
$$

for all $x \in h^{-1}(K)$ and all sufficiently small $\varepsilon$.

Define two sequences of stopping times:

$$
\sigma_{0}^{x, \varepsilon}=0 ; \quad \tau_{n}^{x, \varepsilon}=\inf \left\{t \geq \sigma_{n-1}: z_{t}^{x, \varepsilon}=0\right\}, n \geq 1 ; \quad \sigma_{n}^{x, \varepsilon}=\inf \left\{t \geq \tau_{n}:\left|z_{t}^{x, \varepsilon}\right|=\delta\right\}, n \geq 1,
$$

where $\delta>0$ will be selected later. Then

$$
\begin{gathered}
\mathrm{E}\left(f\left(Y_{T}^{x, \varepsilon}\right)-f\left(Y_{0}^{x, \varepsilon}\right)-\int_{0}^{T} A f\left(Y_{t}^{x, \varepsilon}\right) d t\right)= \\
\mathrm{E} \sum_{n=1}^{\infty}\left(f\left(Y_{\tau_{n}^{x, \varepsilon} \wedge T}^{x, \varepsilon}\right)-f\left(Y_{\sigma_{n-1}^{x, \varepsilon} \wedge T}^{x, \varepsilon}\right)-\int_{\sigma_{n-1}^{x, \varepsilon} \wedge T}^{\tau_{n}^{x, \varepsilon} \wedge T} A f\left(Y_{t}^{x, \varepsilon}\right) d t\right)+ \\
\mathrm{E} \sum_{n=1}^{\infty}\left(f\left(Y_{\sigma_{n}^{x, \varepsilon} \wedge T}^{x, \varepsilon}\right)-f\left(Y_{\tau_{n}^{x, \varepsilon} \wedge T}^{x, \varepsilon}\right)-\int_{\tau_{n}^{x, \varepsilon} \wedge T}^{\sigma_{n}^{x, \varepsilon} \wedge T} A f\left(Y_{t}^{x, \varepsilon}\right) d t\right) .
\end{gathered}
$$

In order to control the number of terms in the sums above, we will need the following lemma. 
Averaging in the case of multiple invariant measures for the fast system

Lemma 5.5. There is $c>0$ such that, for all sufficiently small $\delta$,

$$
\mathrm{P}\left(\sigma_{n}^{x, \varepsilon} \leq T\right) \leq \exp (-c \delta n), \quad x \in M, \quad n \geq 2 .
$$

Proof. Let $A_{t}$ be an auxiliary diffusion process, $d A_{t}=a d t+d W_{t}, A_{0}=-\delta$, where $a=\sup _{i, z}|v(i, z)|$. Let $\tilde{\tau}=\inf \left\{t: A_{t}=0\right\}$. Then $\mathrm{P}(\tilde{\tau} \leq T) \leq \exp (-c \delta)$ for some $c>0$. If $\tilde{\tau}_{k}, k \geq 1$, is a sequence of independent random variables distributed as $\tilde{\tau}$, then

$$
\mathrm{P}\left(\tilde{\tau}_{1}+\ldots+\tilde{\tau}_{n} \leq T\right) \leq \exp (-c \delta n)
$$

From the definition of the stopping times and the process $X_{t}^{x, \varepsilon}$ it follows that

$$
\mathrm{P}\left(\tau_{n}^{x, \varepsilon}-\sigma_{n-1}^{x, \varepsilon}>s \mid \mathcal{F}_{\sigma_{n-1}^{x, \varepsilon}}^{x, \varepsilon}\right) \geq \mathrm{P}(\tilde{\tau}>s)
$$

for each $n \geq 2$ and $s \geq 0$. Therefore, estimate (5.8), with $\tau_{n+1}^{x, \varepsilon}$ instead of $\sigma_{n}^{x, \varepsilon}$, follows from (5.9) and the strong Markov property. Thus, the original formula (5.8) also holds, with a different constant $c$.

Let

$$
\alpha(x, n)=\mathrm{E}\left(f\left(Y_{\tau_{n}^{x, \varepsilon} \wedge T}^{x, \varepsilon}\right)-f\left(Y_{\sigma_{n-1}^{x, \varepsilon} \wedge T}^{x, \varepsilon}\right)-\int_{\sigma_{n-1}^{x, \varepsilon} \wedge T}^{\tau_{n}^{x, \varepsilon} \wedge T} A f\left(Y_{t}^{x, \varepsilon}\right) d t \mid \mathcal{F}_{\sigma_{n-1}^{x, \varepsilon} \wedge T}^{x, \varepsilon}\right) .
$$

Observe that

$$
\lim _{\varepsilon \downarrow 0} \sup _{x \in h^{-1}(K)} \sup _{n \geq 1}|\alpha(x, n)|=0
$$

uniformly in all the realizations of the randomness (which is present since we are taking the conditional expectation). This is a standard averaging result for the fast-slow system in the case of a single invariant measure for the fast motion. It easily follows from the explicit construction of $X_{t}^{x, \varepsilon}$. Therefore, for the first expectation in (5.7), by Lemma 5.5, we get

$$
\begin{gathered}
\left|\mathrm{E} \sum_{n=1}^{\infty}\left(f\left(Y_{\tau_{n}^{x, \varepsilon} \wedge T}^{x, \varepsilon}\right)-f\left(Y_{\sigma_{n-1}^{x, \varepsilon} \wedge T}^{x, \varepsilon}\right)-\int_{\sigma_{n-1}^{x, \varepsilon} \wedge T}^{\tau_{n}^{x, \varepsilon} \wedge T} A f\left(Y_{t}^{x, \varepsilon}\right) d t\right)\right| \leq \\
\leq \sum_{n=1}^{\infty}|\alpha(x, n)| \mathrm{P}\left(\sigma_{n-1}^{x, \varepsilon} \leq T\right) \rightarrow 0 \quad \text { as } \varepsilon \downarrow 0,
\end{gathered}
$$

uniformly in $x \in h^{-1}(K)$.

Next, observe that

$$
\left|\mathrm{E}\left(\sigma_{n}^{x, \varepsilon} \wedge T-\tau_{n}^{x, \varepsilon} \wedge T \mid \mathcal{F}_{\tau_{n}^{x, \varepsilon} \wedge T}^{x, \varepsilon}\right)\right| \leq C \delta^{2}
$$

for some constant $C$ and all $x \in M, n \geq 1$. This follows from the fact that the process $z_{t}^{x, \varepsilon}$ is a Brownian motion with a bounded variable drift, and the expectation of its exit time from the $\delta$-neighborhood of the origin is estimated from above by $C \delta^{2}$. Therefore,

$$
\left|\mathrm{E} \sum_{n=1}^{\infty} \int_{\tau_{n}^{x, \varepsilon} \wedge T}^{\sigma_{n}^{x, \varepsilon} \wedge T} A f\left(Y_{t}^{x, \varepsilon}\right) d t\right| \leq C \delta^{2} \sup |A f| \sum_{n=1}^{\infty} \mathrm{P}\left(\tau_{n}^{x, \varepsilon} \leq T\right) .
$$

By Lemma 5.5, since $\tau_{n}^{x, \varepsilon} \geq \sigma_{n-1}^{x, \varepsilon}$, the right hand side does not exceed $K \delta$ for some constant $K$. This is smaller than $\eta / 2$ for all sufficiently small $\delta$. Thus it remains to show that there is $\delta>0$ such that

$$
\left|\mathrm{E} \sum_{n=1}^{\infty}\left(f\left(Y_{\sigma_{n}^{x, \varepsilon} \wedge T}^{x, \varepsilon}\right)-f\left(Y_{\tau_{n}^{x, \varepsilon} \wedge T}^{x, \varepsilon}\right)\right)\right|<\eta / 2
$$


Averaging in the case of multiple invariant measures for the fast system

for all sufficiently small $\varepsilon$. Observe that

$$
\left.\mid \mathrm{E} \sum_{n=1}^{\infty}\left(f\left(Y_{\sigma_{n}^{x, \varepsilon}}^{x, \varepsilon}\right)-f\left(Y_{\sigma_{n}^{x, \varepsilon} \wedge T}^{x, \varepsilon}\right)\right) \chi_{\left\{{ }_{n}\right.}^{x, \varepsilon} \leq T\right\}|\leq \sup | f\left(l_{1}, z_{1}\right)-f\left(l_{2}, z_{2}\right) \mid<\eta / 4
$$

for all sufficiently small $\delta$, where the supremum is taken over all $l_{1}, l_{2}$ and $z_{1}, z_{2}$ such that $\left|z_{1}\right|,\left|z_{2}\right| \leq \delta$. Therefore,

$$
\left|\mathrm{E} \sum_{n=1}^{\infty}\left(f\left(Y_{\sigma_{n}^{x, \varepsilon} \wedge T}^{x, \varepsilon}\right)-f\left(Y_{\tau_{n}^{x, \varepsilon} \wedge T}^{x, \varepsilon}\right)\right)\right| \leq \eta / 4+\sup _{x: h(x)=O} \mathrm{E}\left(f\left(Y_{\sigma}^{x, \varepsilon}\right)-f\left(Y_{0}^{x, \varepsilon}\right)\right) \sum_{n=1}^{\infty} \mathrm{P}\left(\tau_{n}^{x, \varepsilon} \leq T\right),
$$

where $\sigma=\sigma^{x, \varepsilon}=\inf \left\{t \geq 0:\left|z_{t}^{x, \varepsilon}\right|=\delta\right\}$. By Lemma 5.5, since $\tau_{n}^{x, \varepsilon} \geq \sigma_{n-1}^{x, \varepsilon}$, the sum in the right hand side can be estimated from above by $K / \delta$ for some $K$, and it remains to show that $\sup _{x: h(x)=O} \mathrm{E}\left(f\left(Y_{\sigma}^{x, \varepsilon}\right)-f\left(Y_{0}^{x, \varepsilon}\right)\right) / \delta$ can be made arbitrarily small for some $\delta$ and all sufficiently small $\varepsilon$. Since $f(l, z)$ is differentiable in $z$ at $z=0$ along each edge (one-sided derivatives exist), and the relation between the derivatives is given by (3.2) with $\bar{\pi}_{1}, \bar{\pi}_{2}$ defined in (2.3), the result follows from the following lemma.

Lemma 5.6. For each $\eta>0$, for all sufficiently small $\delta>0$,

$$
\left|\mathrm{P}\left(\xi_{\sigma}^{x, \varepsilon} \in R_{1}, z_{\sigma}^{x, \varepsilon}=\delta\right)-\frac{\bar{\pi}_{1}}{2}\right| \leq \eta, \quad\left|\mathrm{P}\left(\xi_{\sigma}^{x, \varepsilon} \in R_{2}, z_{\sigma}^{x, \varepsilon}=\delta\right)-\frac{\bar{\pi}_{2}}{2}\right| \leq \eta
$$

for each $x$ such that $h(x)=O$ and all sufficiently small $\varepsilon$ (depending on $\delta$ ).

Proof. The idea of the proof is as follows. First, using the Girsanov theorem, we replace the original process with a similar one but without drift. The slow component of the process without the drift is a Brownian motion that does not depend on the fast variable. Then, for almost every realization of the Brownian motion, the fast component is a time-inhomogeneous Markov chain, whose state space can be separated into two classes ( $R_{1}$ and $R_{2}$ ) that communicate when the Brownian motion is to the left of the origin and don't communicate when the Brownian motion is to the right of the origin. Almost immediately, the distribution of the fast component will become close to $\pi$ due to the times that the Brownian motion spends on the negative semi-axis. The probability that the Brownian motion exits the interval $[-\delta, \delta]$ through the right end point is $1 / 2$, while the probability that the fast component is in $R_{1}\left(R_{2}\right)$ at that time is close to $\bar{\pi}_{1}\left(\bar{\pi}_{2}\right)$, giving the desired result. Let us make these arguments more rigorous.

Consider an auxiliary process $\tilde{X}_{t}^{x, \varepsilon}=\left(\tilde{\xi}_{t}^{x, \varepsilon}, \tilde{z}_{t}^{x, \varepsilon}\right)$ that is defined the same way as $X_{t}^{x, \varepsilon}$, but with $v(i, \cdot) \equiv 0$ for each $i$. The corresponding stopping time will be denoted by $\tilde{\sigma}$. Let $\tilde{\mu}_{t_{0}}$ and $\mu_{t_{0}}$ be the measures on the space of RCLL functions from $\left[0, t_{0}\right]$ to $M$ induced by the processes $\tilde{X}_{t}^{x, \varepsilon}$ and $X_{t}^{x, \varepsilon}$, respectively. By the Girsanov theorem, $\tilde{\mu}_{t_{0}}$ and $\mu_{t_{0}}$ are mutually absolutely continuous. Moreover, for each $\eta>0$, for all sufficiently small $t_{0}$ and $\varepsilon$, we have $\tilde{\mu}_{t_{0}}\left(1-\eta \leq p_{t_{0}} \leq 1+\eta\right) \geq 1-\eta$ for each $x=(i, 0)$, where $p_{t_{0}}$ is the density of $\mu_{t_{0}}$ with respect to $\tilde{\mu}_{t_{0}}$. Since $\mathrm{P}\left(\tilde{\sigma} \leq t_{0}\right) \rightarrow 1$ as $\delta \downarrow 0$, we have, for all sufficiently small $\delta$ and $\varepsilon$ and $x=(i, 0)$,

$$
\lim _{\delta \downarrow 0}\left|\mathrm{P}\left(\xi_{\sigma}^{x, \varepsilon} \in R_{1}, z_{\sigma}^{x, \varepsilon}=\delta\right)-\mathrm{P}\left(\tilde{\xi}_{\tilde{\sigma}}^{x, \varepsilon} \in R_{1}, \tilde{z}_{\tilde{\sigma}}^{x, \varepsilon}=\delta\right)\right| \leq \frac{\eta}{2} .
$$

Similarly,

$$
\lim _{\delta \downarrow 0}\left|\mathrm{P}\left(\xi_{\sigma}^{x, \varepsilon} \in R_{2}, z_{\sigma}^{x, \varepsilon}=\delta\right)-\mathrm{P}\left(\tilde{\xi}_{\tilde{\sigma}}^{x, \varepsilon} \in R_{2}, \tilde{z}_{\tilde{\sigma}}^{x, \varepsilon}=\delta\right)\right| \leq \frac{\eta}{2} .
$$

Thus it is sufficient to prove Lemma 5.6 in the case when there is no drift term.

The process without the drift term can be constructed as follows. For $x=(i, z) \in M$, let $\tilde{z}_{t}^{x}$ be a one-dimensional Brownian motion starting at $z$ (the slow variable does not 
Averaging in the case of multiple invariant measures for the fast system

depend on $\varepsilon$ since there is no drift). This process is defined on a probability space denoted by $\left(\Omega_{1}, \mathcal{F}_{1}, \mathrm{P}_{1}\right)$. Let $\left(\Omega_{2}, \mathcal{F}_{2}, \mathrm{P}_{2}\right)$ be another probability space. For each $\omega_{1} \in \Omega_{1}$, we define $\tilde{\xi}_{t}^{x, \varepsilon}$ to be a time-inhomogeneous Markov process on $\left(\Omega_{2}, \mathcal{F}_{2}, \mathrm{P}_{2}\right)$ with transition rates at time $t$ given by $\varepsilon^{-1} q_{i j}\left(\tilde{z}_{t}^{x}\left(\omega_{1}\right)\right)$. Then $\tilde{X}_{t}^{x, \varepsilon}=\left(\tilde{\xi}_{t}^{x, \varepsilon}, \tilde{z}_{t}^{x}\right)=\left(\tilde{\xi}_{t}^{x, \varepsilon}\left(\omega_{1}, \omega_{2}\right), \tilde{z}_{t}^{x}\left(\omega_{1}\right)\right)$ is the required process if we consider it on the product space $\left(\Omega_{1} \times \Omega_{2}, \mathcal{F}_{1} \times \mathcal{F}_{2}, \mathrm{P}_{1} \times \mathrm{P}_{2}\right)$.

Let $\sigma^{\prime}=\inf \left\{t \geq 0: \tilde{z}_{t}^{x, \varepsilon}=-\delta^{2}\right\}$. By conditioning on the event $\sigma^{\prime}<\tilde{\sigma}$, whose probability tends to one as $\delta \downarrow 0$, we see that it is sufficient to prove

$$
\left|\mathrm{P}\left(\tilde{\xi}_{\tilde{\sigma}}^{x, \varepsilon} \in R_{1}, \tilde{z}_{\tilde{\sigma}}^{x}=\delta\right)-\frac{\bar{\pi}_{1}}{2}\right| \leq \frac{\eta}{2}, \quad\left|\mathrm{P}\left(\tilde{\xi}_{\tilde{\sigma}}^{x, \varepsilon} \in R_{2}, \tilde{z}_{\tilde{\sigma}}^{x}=\delta\right)-\frac{\bar{\pi}_{2}}{2}\right| \leq \frac{\eta}{2}
$$

for each $x=\left(i,-\delta^{2}\right)$. By taking $\delta$ sufficiently small, we can make sure that $\mathrm{P}_{1}\left(\tilde{z}_{\tilde{\sigma}}^{x}=\delta\right)$ is arbitrarily close to $1 / 2$ if $x=\left(i,-\delta^{2}\right)$. Thus (5.10) will follow if we show that

$$
\left|\mathrm{P}_{2}\left(\tilde{\xi}_{\tilde{\sigma}}^{x, \varepsilon} \in R_{1}\right)-\bar{\pi}_{1}\right| \leq \frac{\eta}{4}, \quad\left|\mathrm{P}_{2}\left(\tilde{\xi}_{\tilde{\sigma}}^{x, \varepsilon} \in R_{2}\right)-\bar{\pi}_{2}\right| \leq \frac{\eta}{4}
$$

for each $x=\left(i,-\delta^{2}\right)$, each $\omega_{1}$ such that $\tilde{z}_{\tilde{\sigma}}^{x}\left(\omega_{1}\right)=\delta$, and all sufficiently small $\varepsilon$ (depending on $\delta$ and $\left.\omega_{1}\right)$.

Now fix $\omega_{1}$ and consider $\tilde{\xi}_{t}^{x, \varepsilon}$ as a time-inhomogeneous Markov process on the space $\left(\Omega_{2}, \mathcal{F}_{2}, \mathrm{P}_{2}\right)$. Recall that the time-homogeneous Markov chain $\Xi_{t}^{-\delta^{2}}$ has a unique invariant distribution $\mu\left(-\delta^{2}\right)=\left(\mu_{1}\left(-\delta^{2}\right), \ldots, \mu_{n}\left(-\delta^{2}\right)\right)$. Moreover, by lemma (2.1), $\lim _{\delta \downarrow} \mid \mu_{i}\left(-\delta^{2}\right)-$ $\pi_{i} \mid=0$ for each $i$. Therefore, for each $t>0$, the distribution of $\Xi_{t / \varepsilon}^{-\delta^{2}}$ can be made arbitrarily close to $\pi=\left(\pi_{1}, \ldots, \pi_{n}\right)$, by taking a sufficiently small $\delta$ first and then taking sufficiently small $\varepsilon$. For all sufficiently small $t$, the time-dependent transition probabilities of the process $\tilde{\xi}_{t}^{x, \varepsilon}$ are close to those of the process $\Xi_{t / \varepsilon}^{-\delta^{2}}$. Therefore, the distribution of $\tilde{\xi}_{t \wedge \tilde{\sigma}}^{x, \varepsilon}$ can be made arbitrarily close to $\pi$, by taking a sufficiently small $\delta$ first, then fixing a sufficiently small $t$, and then taking sufficiently small $\varepsilon$.

Thus, using the Markov property, we can replace the initial point $x$ in (5.11) by an initial distribution with the property that the distribution of the first component (which will be denoted by $\nu$ ) is arbitrarily close to $\pi$, while the second component is $z \in[-\delta, \delta]$. Let $M(z)$ be the generator of $\Xi_{t}^{z}$ and $e^{t M(z)}, t \geq 0$, be the corresponding semigroup acting on distributions. It remains to exhibit a sufficiently small neighborhood of $\pi$ that is invariant for $e^{t M(z)}$ for all sufficiently small $|z|$. Let $\alpha_{1}<0$. From the property (2.1) of the transition matrix it follows that we can find $\alpha_{2}>0$ and $\delta>0$ such that, for $t \geq 0$,

$$
\left|\sum_{i \in R_{1}}\left(e^{t M(z)} \nu\right)_{i}-\bar{\pi}_{1}\right| \leq \alpha_{1}, \quad\left|\sum_{i \in R_{2}}\left(e^{t M(z)} \nu\right)_{i}-\bar{\pi}_{2}\right| \leq \alpha_{1},
$$

provided that $|z| \leq \delta$ and the following conditions are satisfied:

$$
\begin{gathered}
\left|\sum_{i \in R_{1}} \nu_{i}-\bar{\pi}_{1}\right| \leq \alpha_{1}, \quad\left|\sum_{i \in R_{2}} \nu_{i}-\bar{\pi}_{2}\right| \leq \alpha_{1}, \\
\left|\frac{\nu_{i}}{\sum_{j \in R_{1}} \nu_{j}}-\pi_{i}\right| \leq \alpha_{2}, \quad i \in R_{1}, \quad\left|\frac{\nu_{i}}{\sum_{j \in R_{2}} \nu_{j}}-\pi_{i}\right| \leq \alpha_{2}, \quad i \in R_{2} .
\end{gathered}
$$

Moreover, by taking $\delta$ smaller, if needed, and assuming that $\alpha_{1}<\min \left(\bar{\pi}_{1}, \bar{\pi}_{2}\right) / 2$, we can make sure that

$$
\left|\frac{\left(e^{t M(z)} \nu\right)_{i}}{\sum_{j \in R_{1}}\left(e^{t M(z)} \nu\right)_{j}}-\pi_{i}\right| \leq \alpha_{2}, \quad i \in R_{1}, \quad\left|\frac{\left(e^{t M(z)} \nu\right)_{i}}{\sum_{j \in R_{2}}\left(e^{t M(z)} \nu\right)_{j}}-\pi_{i}\right| \leq \alpha_{2}, \quad i \in R_{2},
$$

provided that (5.12) and (5.13) hold. Thus the set of distributions $\nu$ satisfying (5.12) and (5.13) for is invariant for $e^{t M(z)}$ if $|z| \leq \delta$.

By our construction, the distribution of $\tilde{\xi}_{\tilde{\sigma}}^{x, \varepsilon}$ (with the $x$ distributed as $(\nu, z)$ ) is $\exp \left(\varepsilon^{-1} \int_{0}^{\tilde{\sigma}} M\left(\tilde{z}_{t}^{x}\left(\omega_{1}\right)\right) d t\right) \nu$. It remains to choose $\alpha_{1}=\eta / 4$.

As we discussed above, Lemma 5.6 completes the proof of the theorem. 
Averaging in the case of multiple invariant measures for the fast system

\section{References}

[1] E. B. Dynkin, Markov Processes, Springer-Velag, Berlin, Heidelberg, New York, 1965.

[2] S. N. Ethier, T. G. Kurtz, Markov processes: characterization and convergence, Wiley Series in Probability and Mathematical Statistics: Probability and Mathematical Statistics. John Wiley and Sons, Inc., New York, 1986. MR0838085

[3] W. Feller, An introduction to probability theory and its applications, Vol. II. Second edition, John Wiley and Sons, New York, 1971. MR0270403

[4] M. I. Freidlin, Functional Integration and Partial Differential Equations, Princeton University Press, 1985. MR0833742

[5] M. I. Freidlin, Thermostat-like perturbations of an oscillator, J. Stat. Phys. 164 (2016), no. 1, pp. 130-141. MR3509050

[6] M. I. Freidlin, On stochastic perturbations of dynamical systems with a "rough" symmetry. Hierarchy of Markov chains, J. Stat. Phys. 157 (2014), no. 6, 1031-1045. MR3277758

[7] M.I. Freidlin, Long-time influence of small perturbations and motion on the simplex of invariant probability measures, to appear in Pure and Applied Functional Analysis.

[8] M. I. Freidlin, L. Koralov, Metastable Distributions of Markov Chains with Rare Transitions, J Stat Phys 167, 1355-1375 (2017). https://doi.org/10.1007/s10955-017-1777-z MR3652517

[9] M. I. Freidlin, A. D. Wentzell, Random Perturbations of Dynamical Systems, Springer 2012. MR2953753

[10] V. S. Korolyuk, N. I. Portenko, A. V. Skorokhod, A. F. Turbin, Handbook on probability theory and mathematical statistics, Nauka, 1985, (in Russian). MR0727478

[11] P. Mandl, Analytical Treatment of One-dimensional Markov Processes, Springer-Verlag, 1968. MR0247667

Acknowledgments. While working on this article, L. Koralov was supported by the ARO grant W911NF1710419, the University of Maryland Research and Scholarship Award, and the Simons Fellowship (award number 678928). 


\section{Electronic Journal of Probability Electronic Communications in Probability}

\section{Advantages of publishing in EJP-ECP}

- Very high standards

- Free for authors, free for readers

- Quick publication (no backlog)

- Secure publication $\left(\mathrm{LOCKSS}^{1}\right)$

- Easy interface (EJMS²)

\section{Economical model of EJP-ECP}

- Non profit, sponsored by $\mathrm{IMS}^{3}, \mathrm{BS}^{4}$, ProjectEuclid ${ }^{5}$

- Purely electronic

\section{Help keep the journal free and vigorous}

- Donate to the IMS open access fund ${ }^{6}$ (click here to donate!)

- Submit your best articles to EJP-ECP

- Choose EJP-ECP over for-profit journals

\footnotetext{
${ }^{1}$ LOCKSS: Lots of Copies Keep Stuff Safe http://www. lockss.org/

${ }^{2}$ EJMS: Electronic Journal Management System http://www.vtex.lt/en/ejms.html

${ }^{3}$ IMS: Institute of Mathematical Statistics http://www.imstat.org/

${ }^{4}$ BS: Bernoulli Society http://www. bernoulli-society.org/

${ }^{5}$ Project Euclid: https://projecteuclid.org/

${ }^{6}$ IMS Open Access Fund: http://www.imstat.org/publications/open.htm
} 